\title{
Allégories de la sexualité dans l'Ovide moralisé
}

\author{
David F. Hult
}

\section{OpenEdition}

Journals

Édition électronique

URL : https://journals.openedition.org/crm/50

DOI : $10.4000 / \mathrm{crm} .50$

ISSN : 1955-2424

Éditeur

Honoré Champion

Édition imprimée

Date de publication : 10 décembre 2002

ISSN : 1272-9752

\section{Référence électronique}

David F. Hult, "Allégories de la sexualité dans l'Ovide moralisé », Cahiers de recherches médiévales [En ligne], 9 | 2002, mis en ligne le 03 septembre 2007, consulté le 15 décembre 2022. URL : http:// journals.openedition.org/crm/50 ; DOI : https://doi.org/10.4000/crm.50

Ce document a été généré automatiquement le 15 décembre 2022.

Tous droits réservés 


\title{
Allégories de la sexualité dans l'Ovide moralisé
}

\author{
David F. Hult
}

1 Source incontournable des mythes ovidiens pour les poètes du XIV et du XV ${ }^{e}$ siècle, tels que Machaut, Froissart et Christine de Pizan, première traduction en langue vernaculaire de l'ensemble des Métamorphoses d'Ovide, l'Ovide moralisé est universellement connu, au moins de nom, par les spécialistes de la littérature médiévale française. Mais il reste un texte peu lu, «parent pauvre » parmi les œuvres $\mathrm{du}$ XIV ${ }^{\mathrm{e}}$ siècle étudiées par la critique, comme le dit si bien M.-R. Jung ${ }^{1}$. Certes, les raisons de cette situation sont compréhensibles : la longueur du poème, quelque 70000 vers octosyllabiques, et son inaccessibilité relative en rendent l'approche pour le moins difficile $^{2}$. Le titre même, qui est de toute évidence une étiquette appliquée au siècle dernier $^{3}$, a tout pour rebuter un public moderne, en ce qu'il suggère un didactisme, voire un type de prédication qui est de nos jours peu à la mode. Mais ce que nous allons essayer de montrer dans les pages qui suivent, c'est que le type de lecture qui ferait de ce texte une œuvre d'édification pure et simple néglige une polémique sous-jacente mais inhérente au projet même de traiter de la métamorphose, polémique qui déstabilise la relation présumée hiérarchique entre texte et glose, fable et sentence.

2 L'Ovide moralisé, œuvre anonyme, fut composé au premier quart du XIV ${ }^{e}$ siècle, certainement avant 1328 et peut-être aussi tôt que $1315^{4}$. Son auteur, plus ou moins contemporain de Dante (mort en 1321) et de la génération suivant Jean de Meun (mort en 1305), parle ouvertement de son entreprise, mais se tait sur ce qui concerne sa propre identité et les raisons précises pour lesquelles il a composé sa traduction. Sa connaissance du latin et de la tradition abondante de commentaires ovidiens, ses explications basées sur la cosmologie et la théologie, suggèrent soit qu'il était moine, soit qu'il était lié au monde universitaire, comme c'était le cas pour Jean de Meun. Dans son épilogue, il fait une référence en passant aux lecteurs qu'il envisageait :

Emprez je pri tous les lectors,

Tous les maistres, tous les rectors

Qui orront et liront ce dit

Que, s'il y a vice ou mesdit 
Qui n'i doie estre, ou s'il y fault,

Par oubliance ou par default,

Chose que g'i doie avoir mis,

Come seignors et come amis,

Qu'il sousportent la non sachance,

La faute et la non souffisance

De mon engin, quar je n'ai mie

Tel sens ne tel philosophie

Qu'il n'i puisse avoir à redire

Et c'uns autres n'en peüst dire

Miex assez, s'il l'eüst empris. (OM, XV, vv. 7473-87)

La référence à des recteurs et à des maîtres d'école confirme bien un milieu scolaire, mais suggère que l'auteur occupe une position subalterne, surtout lorsqu'on considère ses protestations d'insuffisance («la non suffisance/de mon engin»), d'un manque d'expérience («moi, le maindre des menors,/Enfant non sachant et novice » [OM, XV, vv. 7432-33] - après avoir écrit 70000 vers!) et son affirmation défensive d'avoir agi selon ce qui est juste et vertueux malgré les mauvaises réactions éventuelles de ses lecteurs. Ainsi, quelques vers plus tard, il s'excuse devant toute personne qui aurait pu se sentir critiquée dans son livre:

Quar ce n'est pas m'entencion

De faire reprehension

Contre nulle sengle persone.

Mes, si com la sentence done

De l'auctor, au plus loiaument

Que je puis, ai generaument

Les vices blasmez et repris,

Sans entente d'avoir mespris

Vers nul home qui soit en vie.

Quar par amours ne par envie

N'en ai nul loé ne blasmé.

Nulz ne s'en tiegne à diffamé

Pour nulle reprehension,

Quar n'ai eüe entencion

De reprouver ne de remordre

Nulle dignité ne nul ordre

Fors sans plus l'estat de pechié.

Et se nulz s'en sent entechié,

Ament soi, si fera que sage,

Sans haïr m'ent en son corage. (OM, XV, vv. 7501-7520)

4 Ici et ailleurs, il y a toutes chances que le traducteur d'Ovide ait été influencé par Jean de Meun, et on peut tirer profit de la comparaison avec la célèbre apologie du continuateur du Roman de la Rose, qui, lui aussi, base sa défense sur la différence entre une attaque ad hominem, et une critique générale du vice ${ }^{5}$ :

Et se gent contre moi groucent

Qui se tormentent et corroucent,

Qui sentent que je les remorde

Pour ce chapitre ou je recorde

Les paroles de faus samblant,

Et pour ce s'aillent assamblant

Que blamer ou pugnir me vueillent,

Pour ce que de mon dit se dueillent,

Je faz bien protestacion

C'onques ne fu m'entencion 
De parler contre houme vivant

Sainte religion sivant

Ne qui sa vie use en bonne oevre,

De quelque robe qu'il se coevre.

Ainz pris mon arc et l'entesoie,

Quel que pechierres que je soie,

Si fis ma saiete voler

Generalment pour affoler. [...]

Et s'il y a nule parole

Qui sainte eglise tiengne a fole,

Presz sui k'a son voloir l'amende

Se je puis souffire a l'amende.

(Rose, vv. 15247-15264, 15303-15306 ;

je corrige la ponctuation du v. 15252)

5 On entend l'écho du mot " amende ", répété à la rime, dans l'apologie de l'Ovide moralisé que nous venons de citer, écho qui, de manière intéressante, souligne une des différences de perspective cruciales entre ces deux auteurs : là où le discours de Jean de Meun tourne autour d'une justification de soi («Coupes n'en ai ne ja n'avré », Rose, v. 15286), notre traducteur, bien plus modeste, met l'accent sur l'utilité de son écrit: «Diex [...] doint à ce dit tel efficace/Que cil y puissent profiter/Qui l'orront dire et reciter» (OM, XV, vv. 7463-66).

On peut être surpris que notre traducteur vise des clercs dans ces remarques, mais il faut se rappeler que le public susceptible de s'intéresser à des textes en langue vernaculaire, en vers comme en prose, était bien plus large au XIV siècle que même au siècle précédent. Certes, on sait que de nobles seigneurs, peut-être même des bourgeois aisés, furent parmi les premiers patrons, et donc lecteurs, de ce texte ${ }^{6}$, mais le goût pour des textes en français avait également gagné le monde des clercs et des prêtres. Vers 1300 Jean de Meun, dans le prologue de sa traduction de la Consolation de Philosophie de Boèce, mentionne que son ouvrage est destiné à des clercs ainsi qu'à des laïcs, parmi lesquels se situe son patron, le roi Philippe le Bel. Et même pour quelqu'un qui connaît bien le latin, comme celui-ci, «est de moult plus legiers a entendre le françois que le latin $»^{7}$. Les deux langues ont donc un statut distinct mais complémentaire durant les derniers siècles du Moyen Âge. Vers le milieu du siècle, Pierre Bersuire, dans le prologue de son Ovidius moralizatus, parle de l'existence d'une traduction en vers français que Jeanne, autrefois reine de France, avait commandée (fabule poetarum alias fuerant moralizate et ad instantiam illustrissime domine Johane quondam regine francie dudum in rithmis gallicis translate). Il avait reçu le livre des mains de Maître Philippe de Vitry seulement au moment d'avoir terminé son propre livre (quare vero opus ilud non videram quousque tractationem istum penitus perscrisse) et il y trouva quantité de bonnes explications (in quo procul dubio multas bonas exposiciones tam allegoricas quam morales inveni). Enfin, Bersuire avoue avoir revu son propre livre après et précise que, s'il avait omis certaines de ces explications, il les insérait immédiatement dans le sien'. Il est donc fort probable que l'auteur de l'Ovide moralisé, dans son but didactique, visait au moins deux publics: celui des religieux, à la fois prêtres qui pouvaient s'en servir pour étoffer leurs sermons (prononcés en langue vernaculaire depuis au moins un ou deux siècles) et clercs pour qui la lecture d'Ovide pouvait désormais se justifier par son message chrétien sous-jacent; et celui des laïcs qui profiteraient des multiples discours moralisateurs. 
7 C'est précisément ce prétendu aplatissement moralisateur appliqué aux contes fougueux d'Ovide qui continue à rebuter les lecteurs modernes. En effet, la méthode de l'auteur s'assimile nettement à celle utilisée traditionnellement par les exégètes de la Bible. Dans son prologue, l'auteur décrit le principe qui a guidé son approche de ces mythes:

Des le premier comencement Du mont jusqu'a l'avenement Jhesu Christ, qui por nous requerre

Vault descendre du ciel en terre,

Font ci mencion cestes fables, Qui toutes samblent mençoignables, Mes n'i a riens qui ne soit voir :

Qui le sens en porroit savoir,

La veritez seroit aperte,

Qui souz les fables gist couverte. (OM, I, vv. 37-46)

8 Généralement, l'auteur traduit un mythe ou une séquence de mythes, en suivant l'organisation narrative des Métamorphoses, et puis il ajoute une ou même plusieurs gloses interprétatives provenant d'une variété de discours signifiants: historique, scientifique, moral, chrétien. Ensuite, il revient au fil narratif, établissant par là un rythme qui bascule sans arrêt entre mythe et commentaire. Les commentaires établissent une correspondance précise entre les termes du mythe, personnages et actions, et les significations dites "cachées». Le principe même d'une allégorie séculière, que l'on avait développée pendant des siècles pour rendre les auteurs antiques acceptables dans un contexte chrétien, trouve sa justification dans l'idée simple que les fables ou fictions antiques avaient été conçues afin de véhiculer des éléments de doctrine philosophique, morale ou religieuse ${ }^{9}$. En fait, une telle façon de lire était inéluctable pour ceux qui ne voulaient pas risquer l'hérésie, comme notre auteur l'explique :

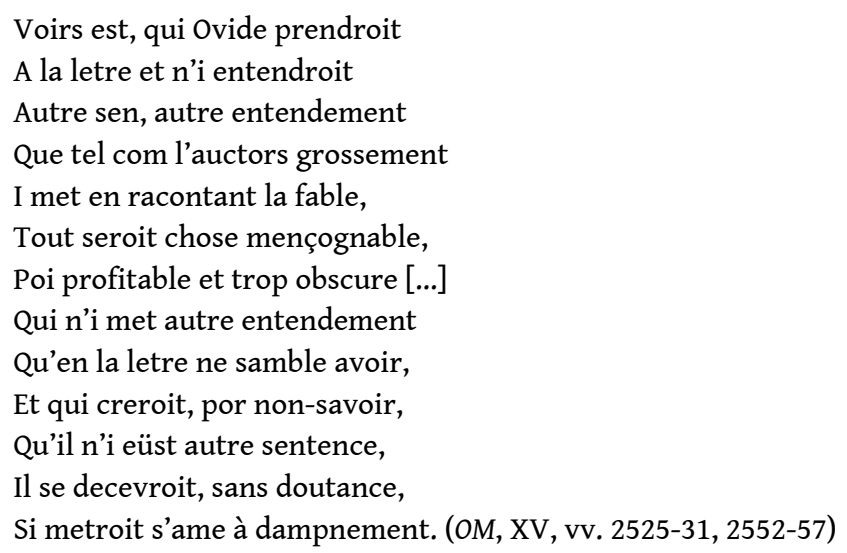

9 D'une part donc, le commentateur doit redresser les mœurs de son public en dégageant des messages pieux de ses textes; d'autre part, sa propre activité comme commentateur est sujette à un jugement moral. Il faut tout de même remarquer que dans les deux cas il ne s'agit point d'éradiquer la lettre mais plutôt de la charger d'une signification autre.

Le contraste avec Jean de Meun est patent, surtout en ce qui concerne l'encadrement religieux. Certes, Jean de Meun connaît bien la tradition des commentaires exégétiques, comme on le détecte aisément dans les remarques de Raison concernant ce qu'elle appelle les allégories des poètes : 
Si dist on bien en nos escoles

Maintes choses par paraboles,

Qui mout sont beles a entendre.

Si ne doit on mie tout prendre

A la lettre quanque l'en ot. [...]

Et qui bien entendroit la lettre

Le sen verroit en escripture

Qui esclarcist la fable oscure.

La verité dedenz reposte

Seroit bele, s'ele ert esposte :

Bien entendras se tu repetes

Les integumenz as poetes. (Rose, vv. 7155-59, 7164-70)

11 On reconnaît bien le même vocabulaire, la même idée que dans l'Ovide moralisé : le sens véritable se trouve caché sous la fable. Mais ce n'est pas là le dernier mot de Jean de Meun, car ces remarques sont placées dans un contexte herméneutique plus large: Raison entre en dialogue avec le narrateur/amant qui, lui, rejette cette autorité en disant qu'il préfère gloser plus tard :

Mais des poetes les sentances,

Les fables ne les methafores

Ne bé je pas a gloser ores. (Rose, vv. 7192-94)

Par ailleurs, cet échange comique, qui finit par traiter la tradition exégétique à la légère, se complète par un manque presque total de commentaires mythographiques traditionnels dans le Roman de la Rose. Là où Jean de Meun joue constamment avec ses lecteurs sur ce sujet, l'auteur de l'ovide moralisé le prend très au sérieux et en fait le principe de son écriture. Un commentateur récent a constaté que «Jean managed to recast the myths'meanings and substitute his own glosses, which emerge from both narrative and his characters'overt interpretations, for those dictated by tradition ", mais on peut également proposer qu'il se débarrasse de la méthode de la glose pour chercher d'autres moyens de produire du sens ${ }^{10}$. En effet, Jean de Meun a souvent été critiqué - on pense notamment à C. S. Lewis - pour son abandon du cadre allégorique de Guillaume de Lorris, pour le fait que les discours de ses personnages commentent directement les mœurs contemporaines. Allant plus loin, A. Minnis a récemment suggéré, et à juste titre, que le genre médiéval auquel appartient la Rose de Jean de Meun est plutôt la satire qui, selon lui, représente le contraire de l'opacité de l'écriture allégorique, car elle critique ouvertement.

13 Si ce n'est pas la méthode allégorique qui relie ces deux textes, en quoi consistent les affinités que l'on détecte entre les deux? Ce sont pour le moins deux textes excessifs, voire extravagants : extravagants dans leur taille volumineuse, dans la multiplicité de leurs gestes interprétatifs, extravagants également dans la place qu'ils laissent à la lubricité et à l'obscénité. Cette dernière extravagance, qui a fait la fortune de Jean de Meun au Moyen Âge et qui n'est que parsemée dans l'Ovide moralisé, tend néanmoins à défaire la hiérarchie communément acceptée dans la relation traditionnelle entre texte et glose, lettre et sens. Or c'est justement une telle suppression mécanique du texte ovidien au profit du message chrétien que l'on a toujours reprochée à l'ovide moralisé. Il suffit de penser aux remarques percutantes de T. Cave, qui résume ainsi : « the model of interpretation [...] moves towards the articulation of a 'hidden sens' not simply in the margins of the original text but in its place». Le texte d'origine est systématiquement déplacé par l'auteur, «while at the same time the range of signification is trapped in what is essentially a monologic discourse $»^{11}$. La substitution monologique, l'acte de 
détention de l'original que Cave voit dans l'ovide moralisé vient compléter les remarques de R. Tuve, dont les écrits sur l'allégorie à la fin du Moyen Âge ont beaucoup influencé la critique anglo-saxonne. Tuve se concentre sur les abus de cette allégorèse (que représente également la moralisation du Roman de la Rose par Jean Molinet) en parlant $\mathrm{du}$ «wild absurdity and irresponsible imposition of equations » typiques de cette tradition ${ }^{12}$. Absurdité, folie, irresponsabilité : S'agit-il réellement des textes médiévaux ou d'une certaine tradition critique qui a toujours eu son parti-pris concernant les normes de l'interprétation?

On peut proposer que l'enjeu de ces deux textes réside plutôt dans le jugement même de ce qui est irresponsable, de ce qui est absurde ou, pour utiliser un terme lourd de sens, moral et linguistique à la fois, de ce qui est propre. La question du propre, dans tous ses sens, est soulevée dans le Roman de la Rose, notamment dans la discussion linguistique de Raison à laquelle nous avons déjà fait allusion. Terme qui désigne la fondation de la dénotation linguistique (la signification littérale d'un mot, par exemple), il s'applique également à son contraire, à tout ce qui se fait pour rendre une chose (ou un mot) acceptable ou honnête, soit par son épuration, soit par son travestissement. Donc, à la fois littera et glose (ou bien euphémisme). Que l'on pense à la définition, fondatrice, que donne Quintilien de la figure de la métaphore, ou translatio ${ }^{13}$ : transfertur ergo nomen aut verbum ex eo loco in quo proprium est, in eum in quo aut proprium deest aut translatum proprio melius est (Institutio oratoria, VIII, vi, 5). [Un nom ou un verbe est transféré de l'endroit auquel il appartient proprement à un autre où il n'y a pas de mot littéral (propre) ou bien lorsque le mot transféré est meilleur que le mot littéral]. Le transfert métaphorique s'opère sur un plan horizontal, à l'intérieur d'un espace linguistique ou textuel uni ; ainsi la métaphore laisse toujours un résidu, car lettre et figure restent en présence. Par contre, l'herméneutique traditionnelle de l'exégèse est toujours conçue de manière verticale, reliant divers plans significatifs au moyen de la substitution. D'où les images de la profondeur, de la pénétration, de la couche, de l'effacement ou du dévoilement pour cerner ce type d'opération rhétorique. Une conséquence importante de cette herméneutique est le rejet du point de départ, du plan initial : s'il faut pénétrer aux profondeurs d'un texte, s'il faut le dévoiler pour en découvrir la vérité, cet acte entraîne nécessairement la destruction de la lettre qui fait obstacle, soit l'emballage jetable de cette vérité.

Cette image traditionnelle de l'exégèse biblique ou païenne se répand non seulement chez les Pères de l'Église, mais également chez des auteurs bien plus proches de l'époque qui nous intéresse, tels que Bernard Sylvestre ou Alain de Lille, qui se servent d'une variété de termes techniques pour parler de l'exégèse des auteurs classiques: termes comme integumentum ou involucrum, qui s'appliquent à une couverture ou à un voile; à des actions comme la séparation du grain de la paille, ou le fait de briser la coque pour savourer la noix cachée à l'intérieur. Mais même dans un texte fondateur tel que le commentaire de Macrobe sur le songe de Scipion, source majeure des théories exégétiques néo-platoniciennes pendant tout le Moyen Âge, l'idée simple de dévoiler la vérité cachée d'un texte païen se trouve mise en question ${ }^{14}$ :

De dis autem (ut dixi) ceteris et de anima non frustra se nec ut oblectent ad fabulosa convertunt, sed quia sciunt inimicam esse naturae apertam nudamque expositionem sui, quae sicut vulgaribus hominum sensibus intellectum sui vario rerum tegmine operimentoque subraxit, ita a prudentibus arcana sua voluit per fabulosa tractari [...] Numenio denique inter philosophos occultorum curiosiori offensam numinum, quod Eleusinia sacra interpretando vulgaverit, somnia prodiderunt, viso sibi ipsas Eleusineas deas 
habitu meretricio ante apertum lupanar videre prostantes, admirantique et causas non convenientis numinibus turpitudinis consulenti respondisse iratas ab ipso se de adyto pudicitiae suae vi abstractas et passim adeuntibus prostitutas. (Macrobius, Comm., I.2. 17-19)

[Mais lorsqu'il s'agit de traiter des autres dieux ou de l'âme, [les philosophes] ont recours aux récits fabuleux, non sans raison, ni simplement pour divertir, mais parce qu'ils savent qu'une exposition ouverte et nue de la Nature lui est fâcheuse, elle qui, de même qu'elle a soustrait aux sens vulgaires des hommes la connaissance d'elle-même au moyen de ses multiples vêtements, voulut que ses secrets soient traités par des hommes sages dans des récits fabuleux [...] En effet, on révéla à Numenius, parmi les plus curieux des philosophes en ce qui concerne les choses occultes, dans un songe l'offense qu'il avait commise envers les dieux lorsqu'il divulgua les secrets Éleusiniens. Les déesses Éleusiniennes elles-mêmes lui parurent habillées comme des courtisanes, se tenant debout devant un bordel ouvert, et lui, étonné, demanda la raison de cette disgrâce qui ne leur convenait point. Fâchées, elles répondirent qu'il les avait forcées à quitter leur sanctuaire de pudicité et les avait prostituées à tout un chacun.]

16 À travers ce qu'on peut appeler une allégorie de l'expression allégorique, Macrobe suggère qu'une exposition philosophique purement nue ou manifeste n'existe pas, en même temps que son choix d'une image lubrique pour l'exprimer met l'accent sur l'aspect purement charnel de ces récits fabuleux, des gestes dont on se sert pour les pénétrer, et peut-être même des messages qu'ils véhiculent.

17 Que l'on considère dans cette optique les images tactiles et sensuelles, voire érotiques, qui pullulent dans l'Ovide moralisé, mais qui sont censées désigner leur contraire, des idées abstraites et incorporelles : s'agit-il bien d'un monde de choses concrètes dont la mise à l'écart s'impose afin de pouvoir contempler les vérités spirituelles cachées endessous, ou ne faut-il pas plutôt y détecter une certaine ambiguïté indélébile, un commentaire qui n'arrive pas totalement à effacer la chair nue des fables païennes ? Les questions qui se posent sont les suivantes : peut-on continuer à croire à la hiérarchie communément admise entre lettre et sens, ainsi qu'au mouvement de l'exégèse qui procède toujours dans un sens unique? La fable disparaît-elle une fois le message extrait? Peut-on détecter dans l'Ovide moralisé une série de leurres qui le rapprochent des jeux linguistiques manifestes d'un Jean de Meun?

Le mécanisme de ce jeu linguistique chez Jean de Meun, dont on a déjà vu certains aspects dans le discours de Raison, se formule nettement dans un passage enfoui au milieu de la longue confession de Nature :

Et pour fere entendre la chose

Bien en puet on, en lieu de glose,

A brief mot .i. example metre

Pour mieus faire esclaircir la letre. (Rose, vv. 16855-58)

Comme dans une rétractation (ou bien un raffinement) de ce qu'avait dit son double allégorique, Raison, Nature semble déclarer qu'une glose et un exemple sont distincts, ou au moins qu'ils fonctionnent différemment : elle prétend substituer celui-ci à celle-là pour mieux éclairer le sens littéral. Ainsi elle préconise une clarification qui ne s'effectue pas par un commentaire exégétique, que Raison désigne par l'expression «integumenz as poetes», mais ce n'est pas non plus selon l'autre option offerte par Raison, une explication «a la lettre/Tout proprement sanz glose mettre» (Rose, vv. 7185-86). Ni l'un, ni l'autre; c'est en effet le travail de la métaphore que propose Nature. Cette remarque assez brève se trouve au milieu d'une discussion que l'on peut 
dire pour le moins peu scrutée par la plupart des lecteurs de la Rose : l'explication des taches d'ombre à la surface de la lune. Ayant décrit le phénomène, Jean de Meun offre son "exemple", qui consiste en l'image de la lumière qui passe par un verre transparent, par contraste avec de la lumière réfléchie par une surface polie ou opaque. Les taches sur la lune s'expliquent analogiquement par la différence optique entre ces deux phénomènes. Mais il ne faut pas oublier que dans la tradition exégétique, comme pour Jean de Meun, les images de la lumière et de l'ombre, du miroir, de l'opacité, sont toutes liées à l'idée de la compréhension ou même de l'intellection. Comme on l'a souvent remarqué par ailleurs, Jean dote le poème commencé par Guillaume de Lorris d'un nouveau nom, le Mirouer aus amoureus. De même, Quintilien, auteur sans doute peu connu au Moyen Âge, fait une analyse très suggestive de la métaphore qui suit immédiatement le passage que nous venons de citer :

Quae quidem cum ita est ab ipsa nobis concessa natura, ut indocti quoque ac non sentientes ea frequenter utantur, tum ita iucunda atque nitida, ut in oratione quamlibet clara proprio tamen lumine eluceat. (Inst. Or. VIII, vi, 4)

[(La métaphore) ne se recommande pas seulement à nous parce que, de sa propre nature, même les gens incultes ou ceux qui n'y font pas attention s'en servent fréquemment, mais elle est si agréable et si raffinée que, quelle que soit la distinction du discours où elle se trouve, elle brille d'une lumière qui est toute à elle.]

Or, d'après l'exemple plutôt paradoxal de Jean de Meun, la surface opaque brille tandis que la transparence produit de l'obscurité. En effet, l'exemple optique offre une analogie visuelle avec l'exemplarité même et suggère, avec Quintilien, que la lumière la plus pénétrante se trouve dans le langage métaphorique rayonnant d'un texte qui, comme la lune, est " par lieus oscure» (Rose, v. 16842).

21 Le fonctionnement de ce jeu métaphorique chez Jean de Meun est souvent lié à des images charnelles, sinon franchement érotiques. J'ai pu montrer ailleurs comment, dans le discours de Raison, une suite d'euphémismes proposés pour parler du sexe masculin se retrouve ailleurs dans le poème, même dans des contextes qui lui sont totalement étrangers. Cela peut servir de point de départ à son humour mordant, mais c'est également une façon d'associer des systèmes sémiotiques totalement différents ${ }^{15}$. Cela peut également affecter notre exégèse des mythes, surtout lorsque, comme c'est souvent le cas chez Jean de Meun, il n'y a pas de glose explicite. Un petit exemple suffira pour illustrer ce phénomène: l'histoire ovidienne de Pygmalion, que Jean raconte vers la fin de sa continuation, a suscité de nombreuses interprétations. La critique a démontré comment ce mythe établit la relation entre le désir sexuel et l'élan créateur de l'artiste; ce serait un modèle non-solipsiste de la procréation et du désir de l'autre qui sert de contre-exemple au Narcisse de Guillaume de Lorris. Pourtant, bien qu'il amplifie le récit d'Ovide, Jean de Meun n'ajoute pas de glose. Un des aspects les plus frappants de ses ajouts consiste en une longue liste des vêtements et parures dont Pygmalion habille sa statue. Au milieu de ce passage nous lisons le détail suivant :

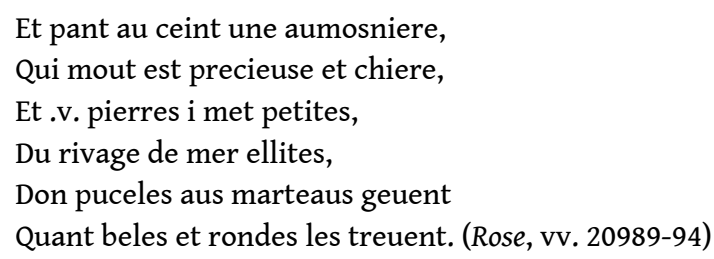

Les éditeurs de la Rose avouent tous qu'ils n'ont pas compris ce passage ${ }^{16}$. Pourtant, il aurait suffi de bien lire le poème de Jean de Meun car, si on place ce passage à côté de 
deux autres qui l'entourent dans le texte, la suggestion devient assez transparente. Genius prononce l'anathème suivant au milieu de sa condamnation des sodomites :

[...] puissent il perdre

Et l'aumosniere et les estalles

Dont il ont signe d'estre malles !

Perte leur viegne des pendanz

A coi l'aumosniere est pendanz!

Les martiaus dedenz estachiez

Puissent il avoir esrachiez! (Rose, vv. 19670-76)

Ensuite, au milieu de la célèbre scène finale de la Rose, nous lisons une description des attributs du narrateur-pèlerin au moment où il s'approche du château et juste avant qu'il consomme de façon allégorique l'acte sexuel :

L'escharpe est de bonne faiture,

D'une pel souple sanz cousture ;

Mais sachiez qu'el n'ere pas vuide :

.Ij. martelez par granz estuide [...]

Nature, qui les me bailla, [...]

Soutilment forgiez i avoit. (Rose, vv. 21361-64, 21367, 21369)

D'une part, ces passages offrent la glose de certains des termes, notamment "aumosniere» et "martiaus», utilisés dans le passage de Pygmalion, termes qui comportent donc bien sûr un sens grivois. En plus, les «martelez» sont le don de Nature, principe même de la procréation, voire de toute création matérielle (on se rappelle que lorsque nous rencontrons Nature, elle se trouve à sa forge, elle qui «Touz jourz martele, touz jorz forge,/Touz jourz ses pieces renouvele/Par generacion nouvele» [Rose, vv. 16014-16]). Ceci dit, l'intention de Jean de Meun reste fort ambiguë, ce qui n'est pas surprenant étant donné la structure dialogique de son ouvrage et le manque de commentaires explicites. Ce que l'on peut affirmer tout de même, c'est qu'il a décidé d'inclure, au milieu de ce volumineux catalogue, suivi d'une énumération d'instruments musicaux, eux aussi chargés de significations sexuelles, l'insinuation que Pygmalion avait doté sa statue bien-aimée d'attributs anatomiques masculins («signe d'estre malle[s]»), d'autant plus surprenant qu'elle suit de peu les harangues de Genius contre les actes homosexuels et sa dénonciation de cet autre personnage ovidien, Orphée. Pour le moins, ce détail atténue la conclusion bien trop facile qui fait de Pygmalion l'exemple même de l'artiste (pro)créateur.

Bien sûr, l'Ovide moralisé fonctionne autrement, car c'est une œuvre mythographique à part entière, ce qui fait que le jeu s'opère à travers la prolifération des gloses plutôt que dans l'introduction de détails suggestifs, comme c'est le cas pour Jean de Meun. Ce qui les relie, pourtant, c'est le vacillement qui se détecte entre la lettre de la fable et l'interprétation mystique ou moralisatrice qui se superpose. En effet, il y a certains aspects de ce texte qui choquent, eu égard à la situation et au but didactique exprimé par l'auteur, et qui valent donc la peine d'être signalés. D'abord, celui-ci, loin d'éviter les nombreuses fables qui ne comportent guère de leçon morale mais qui, par contre, tournent autour de situations scandaleuses ou perverses, semble s'y complaire : inceste, homosexualité, bestialité et ainsi de suite. Point de censure. Au contraire, l'auteur va même plus loin car il insère certains mythes provenant d'autres sources, y compris le récit choquant de l'amour de Pasiphaé pour le taureau, dont le résultat est la naissance du Minotaure. 

fables ovidiennes. La variété de ces interprétations est bien plus large que ce que l'on prétend d'habitude. Les interprétations moralisatrices occupent bien sûr une place importante; la plupart suivent directement le message plus ou moins évident de la fable, par exemple l'orgueil excessif dans le cas de Narcisse, complétées par un grand nombre d'interprétations spécifiquement chrétiennes. Mais l'auteur de l'Ovide moralisé, grand connaisseur des commentaires latins sur Ovide, inclut quantité d'explications naturelles et scientifiques (assimilation des Dieux à des planètes, commentaires météorologiques, etc.). Pourtant, ce qui frappe, ce sont les moments où l'interprétation, plutôt que de passer du littéral à l'abstrait - geste typique d'une interprétation morale ou mystique -,va dans le sens inverse, détectant dans la fable une version plus concrète de son intrigue. Tel est le cas pour les interprétations évhéméristes, c'est-à-dire celles qui interprètent les fables des Dieux comme des histoires de mortels divinisés après leur mort, généralement des hommes politiques importants. Autrement dit, c'est une interprétation qui montre non seulement comment la fable est un récit à interpréter allégoriquement mais comment elle est déjà le résultat d'une opération interprétative. Ainsi, l'interprétation évhémériste du récit de Jupiter et Ganymède : il s'agit d'un roi qui, étant tombé amoureux d'un jeune garçon, le ramène chez lui et en jouit à plusieurs reprises («maintes fois») et «contre nature »; il fait du garçon son bouteiller contre la volonté de sa femme. L'auteur n'y ajoute aucune moralité. La glose de l'histoire d'Iphis est même plus étonnante. Selon Ovide, Iphis, une fille, est élevée comme un garçon par sa mère, car le père avait dit avant sa naissance qu'il tenait à avoir un fils. Le nom Iphis lui-même sert à dissimuler son secret, car il n'est marqué ni par le féminin ni par le masculin en latin; la fille porte des vêtements masculins jusqu'au jour de ses noces. Grâce à une prière à la déesse Isis au dernier moment, juste avant la cérémonie, Iphis est transformée en un homme et les deux époux vivent dans le bonheur. Or, l'auteur de l'Ovide moralisé, tout en écartant une solution sans doute trop féerique à son goût, s'intéresse beaucoup à l'aspect purement concret de la situation. Par conséquent, sa première glose, «selonc historial sentence», prétend qu'une personne comme Iphis a pu réellement exister : «Estre pot », c'est-à-dire, il se peut bien qu'autrefois il y ait eu une femme qui avait les vêtements et les manières d'un homme («D'abit et de cultivement/Sambloit home ", OM, IX, vv. 3118-19). Une autre femme tombe amoureuse de celle-ci, qui, malgré le fait qu'elle n'a pas de quoi consommer leur relation («Tout n'eüst elle point de vit/Ne de membre à ce convenable », OM, IX, vv. 3130-31), l'épouse parce qu'elle désire faire l'amour avec elle. Elle y réussit enfin, grâce à une sorcière, "une orde vilz maquerele»(OM, IX, v. 3143), qui la pourvoit d'un membre artificiel («Par membre apostis», OM, IX, v. 3149) pour tromper sa nouvelle femme. Ici, notre auteur pieux invente une scène qui aurait dû pourtant lui paraître abominable pour expliquer une histoire de transformation sexuelle incroyable mais dont la solution offre une conclusion 'normale', c'est-à-dire, acceptable selon les normes de cette société.

Une troisième observation concerne la mise en œuvre même de l'apparat exégétique. Comme on l'a souvent remarqué, notre auteur, suivant en cela la doctrine des niveaux multiples dans l'exégèse biblique, offre souvent plusieurs gloses l'une après l'autre pour un mythe - deux, trois, même quatre. Mais là où on s'attendrait à une valorisation stable des personnages mythiques à travers les gloses, c'est-à-dire à une exemplarité inhérente à ceux-ci, c'est loin d'être le cas. Deux fables qui traitent de l'inceste illustrent bien cet aspect de la vision interprétative. Myrrha, qui a trompé son père

Cahiers de recherches médiévales, 9 | 2002 
pour pouvoir coucher avec lui, finit par devenir enceinte et donne naissance à Adonis après avoit été transformée en un arbre. L'Ovide moralisé interprète cette dernière d'abord comme la Vierge Marie, qui a conçu Jésus avec Dieu le Père. Ensuite, c'est l'«ame pecheresse » qui, de manière hypocrite, et sans s'être confessée, avale l'hostie, la chair du Christ, le dimanche de Pâques. L'histoire de Biblis offre un autre exemple: celle-ci tombe amoureuse de son frère, que l'auteur de l'ovide moralisé appelle Cadmus ; dans l'histoire ovidienne, Biblis déclare son amour et, son frère, scandalisé, s'étant enfui, elle verse un torrent de larmes, à tel point qu'elle se transforme en une fontaine. Or la première glose, dénommée « istorial ", suit le type d'interprétation rationalisante que nous venons de voir: il se peut que Biblis, amoureuse de son frère, lui ait fait prendre la fuite; par la suite, ne pouvant plus maîtriser ses passions, elle

[...] s'abandona par putage

A touz homes comunement.

Chascuns habandoneement

Pooit en lui puisier et prendre,

Sans contredire et sans deffendre,

Ainsi com l'en puise en fontaine. (OM, IX, vv. 2544-49)

Selon la seconde interprétation, celle-ci «mieudre et plus saine», Cadmus représente «l'umain lignage» et Biblis «divine sapience». La passion que conçoit Biblis pour Cadmus correspond au désir de la divinité de s'unir « contre nature/Charnelment » (OM, IX, vv. 2608-9) à l'homme. On voit bien dans les deux cas la façon dont certains détails du récit d'origine servent à ancrer le rapport avec la glose. Le détail selon lequel Biblis envoie d'abord une lettre à son frère annonçant son désir coupable se compare aux révélations données à Moïse mais que finalement l'humanité n'écoute pas ; sa rencontre ultime est alors assimilée à l'Avènement du Christ, dont la fin est bien sûr la Crucifixion, qui fait de Lui «la fontaine [...]/Dont sourt la pardurable vie». Le passage qui suit cette remarque développe longuement l'image de la fontaine telle qu'elle s'applique à Dieu et au mystère de la Trinité.

Même à l'intérieur du récit chrétien, ou plutôt dans le rapport qui s'établit entre celuici et les histoires d'amour, de séduction, d'accouplement et de métamorphose, il y a un effet de contamination qu'il est difficile de négliger. Dans son prologue, notre auteur nous dit qu'il va ouvrir (" esclorrai»), c'est-à-dire expliquer, «les mutacions des fables » (OM, I, v. 53). Cette expression s'avère bien ambiguë, car les mutacions peuvent faire référence aux métamorphoses proprement dites, les changements corporels, ou aux changements que subissent les fables au cours du travail de l'exégète. Si la métamorphose désigne des corps qui se transforment, elle s'applique également au fonctionnement de l'allégorie, qui transforme le littéral en figuré, la chair en esprit. Pour l'auteur de l'Ovide moralisé, la métamorphose résume toutes ces choses, le principe de la fable ovidienne, du récit, de la glose, mais également le principe même de la vie, comme lorsque deux semences s'unissent dans la matrice. La polyvalence de ce concept, ou plutôt ce concept qui se traduit par la polyvalence, devrait nous faire repenser la prétendue univocité de la démarche exégétique de notre auteur.

Le récit fondateur du christianisme est lui aussi profondément centré sur la relation entre la chair et l'âme, qui se résume en partie dans le mystère de l'Incarnation. Si plusieurs fables ovidiennes offrent des véhicules intrigants, mais souvent étonnants, pour la formulation de ce mystère, elles ne sont pas sans exercer un effet de contamination car elles révèlent de manière oblique tout ce qui est pervers, ou pour 
emprunter l'expression de notre auteur, non naturel, à propos des configurations sexuelles du christianisme. Considérons, après tout, quelques unes de ces relations «familiales » : un père dont le fils est né de sa fille; une fille qui donne naissance à son père; trois personnages, tous masculins, qui s'unissent en une personne, et dont l'un coule de l'autre comme coule l'eau d'une fontaine en une rivière ; enfin, un père qui luimême donne naissance à un fils. L'auteur de l'Ovide moralisé accentue le rapport entre le christianisme et les fables ovidiennes les plus scabreuses lorsqu'il déclare que la naissance du Christ est elle-même "contre nature", expression dont il se sert également pour parler de l'avortement, de la bestialité, comme dans l'histoire de Pasiphaé, de l'homosexualité, comme pour Orphée ou Ganymède, ou de l'inceste, comme c'est le cas, on l'a vu, pour Myrrha et Biblis. Jean de Meun avait déjà dramatisé ce concept fondamental de la théologie chrétienne lorsqu'il fait Dame Nature avouer sa propre incompétence dans cette affaire :

[...] mi sires la mort reçut

Quant il sanz moi prist char humaine

Pour le chaitif oster de paine.

Sanz moi, car je ne sai commant,

Fors qu'il puet tout par son commant.

Ainz fui trop forment esbahie

Quant il de la virge marie

Fu por le chaitif en char nez,

Et puis penduz tout encharnez

Car par moi ne puet ce pas estre

Que riens puisse de virge nestre.(Rose, vv. 19156-66)

L'auteur de l'Ovide moralisé se sert à plusieurs reprises et dans une variété de contextes d'un terme ayant trait à des accouplements spirituels et physiques, voire à la cohésion même du monde : jointure, mot qui est tout aussi polyvalent que celui que l'on vient de voir, mutacion. Ce mot, ou une forme du verbe joindre, s'applique à la montagne de Chaos avant la Création («discordable jointure »), à l'union du corps et de l'âme, du mâle et de la femelle, d'Hermaphrodite à Salmacis, deux corps réunis en un (mais de manière non sexuelle et qui forme un être littéralement ambigu), et enfin à l'Incarnation de Jésus qui s'est uni à la nature humaine. Le concept de jointure s'assimile à celui de mutacion lorsqu'il s'agit de la naissance, deux semences qui s'unissent pour produire un seul être. Mais il s'applique notamment à cette union divine qui, on l'a vu, est par définition contre Nature. Cette association soulève néanmoins certains problèmes parce que, implicitement, elle suggère qu'il y a un rapport nécessaire entre le mystère le plus profond du christianisme et des rapports sexuels jugés abominables. Pour revenir à l'idée évoquée plus haut, que la couverture inquiétante de la fable ovidienne ne disparaît pas totalement une fois le sens profond révélé, se peut-il que les mystères chrétiens, que les conceptions théologiques inintelligibles, ne puissent être conçus qu'à travers ces mêmes fables qui ont tellement fasciné notre auteur?

En guise de conclusion, j'aimerais bien revenir sur quelques autres mythes qui jettent de la lumière sur la discussion précédente. Orphée occupe une position centrale dans le texte d'Ovide car il est promu au statut de narrateur interne au cours du livre X des Métamorphoses. On se rappelle que, après avoir perdu Eurydice, Orphée revient au monde et se fait poète; sa tristesse profonde l'incite à fuir les femmes pour des compagnons masculins. L'association entre Orphée et la sodomie était bien établie à travers le Moyen Âge, comme on l'a vu, et on en trouve une condamnation très élaborée dans le discours de Genius dans le Roman de la Rose. Or, malgré une critique 
très nette des mœurs d'Orphée, notre auteur l'interprète régulièrement in bono dans ses allégories, à tel point qu'il devient une figure de Dieu. La fuite des femmes est interprétée, de manière à la fois intrigante et misogyne, comme le dégoût que ressent Dieu envers la nature féminine :

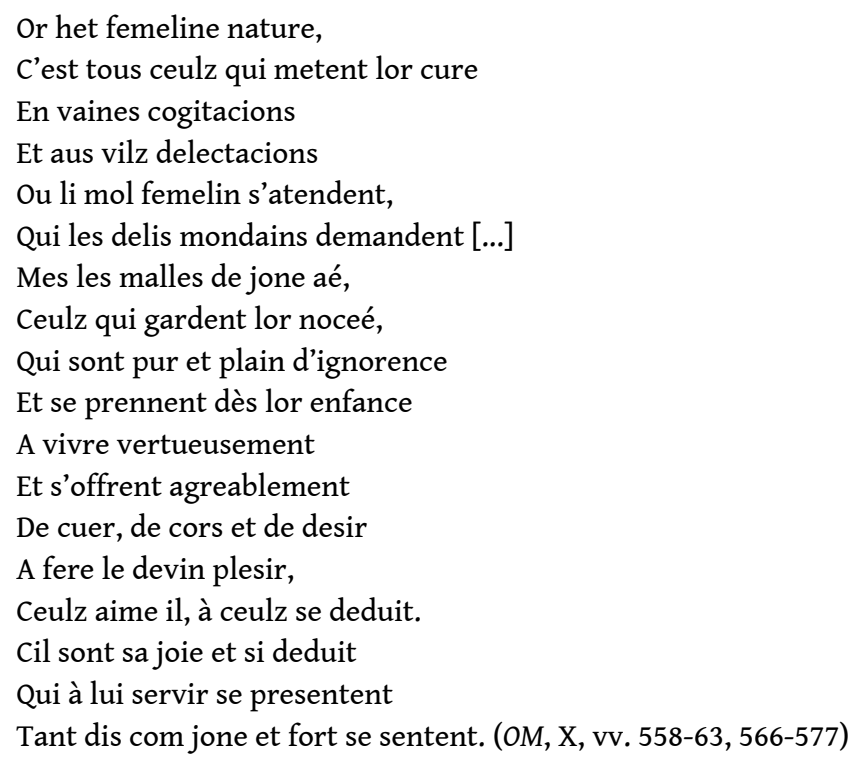

C'est loin d'être la seule critique de la nature féminine dans ce texte: La «meillour sentence » de l'histoire d'Iphis nous révèle que le désir de la part du père d'avoir un fils se traduit par le fait que Dieu méprise "femeline nature», assimilée à «l'ame pecheresse » (OM, IX, vv. 3202-3). La transformation qu'opère Isis à la fin de cette fable, de fille en garçon, n'est rien d'autre que l'efficacité du repentir. De même, dans l'interprétation d'Orphée, Dieu méprise le féminin pour se tourner vers des «malles de jone aé » qui ont gardé leur pureté et leur innocence. Or lorsqu'on parle d'une nature féminine on parle d'une espèce, d'une essence généralisée. Il est donc important de remarquer que la contrepartie de cette nature féminine si méprisée n'est pas le masculin comme espèce, mais des mâles, c'est-à-dire des individus. Le principe des catégories sexuelles (masculin contre féminin) se transforme subtilement en une fantaisie d'accouplements particuliers, l'attirance de Dieu envers les jeunes hommes ${ }^{17}$. Un tel désir, manifestement charnel, est développé également dans la fable de Ganymède, que l'une des gloses interprète comme la chair que Dieu a revêtue pour le rachat de l'humanité: Ganymède, une fois ravi aux cieux comme bouteiller, devient "vrais diex et vrais homs tout ensamble » (OM, X, v. 3421).

En effet, le dégoût que ressent notre auteur pour tout ce qui a trait au féminin, y compris la féminité de certains hommes, suggère que la jointure hétérosexuelle ne peut pas être une image adéquate pour l'Incarnation. Étant donné que le féminin est toujours suspect, qu'il est toujours associé au corps et non à l'esprit, à la chair et non à l'âme (ou, comme dans l'explication d'Iphis, à l'âme pécheresse), c'est un élément déjà imprégné de corruption. Par contre, l'Ovide moralisé semble vouloir étayer la thèse que tout ce qui est purement masculin, y compris ces relations sodomites qui sont par ailleurs indiscutablement condamnées, s'approche du divin. D'où le vif intérêt, surprenant pour un homme d'église, porté vers des formes d'accouplements contre nature qui passe du plus mystique de tous, la conception de Jésus par une vierge, à tout ce qu'il y a de plus concret, le membre artificiel d'Iphis. Même cette dernière histoire, 
imaginée comme une fantaisie lesbienne, finit par illustrer les vertus du masculin au dépens du féminin.

L'auteur était-il conscient des aspects scabreux, pour ne pas dire condamnables, de ces récits et interprétations? On peut répondre par l'affirmative si l'on fait bien attention à son élaboration choquante de l'histoire de Pasiphaé, histoire qui, comme je l'ai dit dans mes remarques préliminaires, a été surajoutée et donc qui reste superflue dans une traduction des Métamorphoses. Pendant la longue description qu'il donne des effets physiques et mentaux de la passion que subit Pasiphaé, le narrateur insère le détail suivant:

[...] De male hore vit

Sa biauté, certes, mes le vit!

Ja certes amé ne l'eüst

Pour biauté, se grant vit n'eüst. (OM, VIII, vv. 765-768)

Vulgarité étonnante pour le moins, mais ensuite le narrateur s'accuse de folie d'une manière qui fait écho à celle de Pasiphaé ainsi qu'à celle d'autres amants ovidiens, rapprochant par là crime de la parole et crime de la chair :

Ha, Diex! Quel honte et quel reprouche !

Comment me vola de la bouche!

Moult me poise qu'il en issi,

Mes à certes ne le dis, si

Eschapa moi ; ne m'en pris garde!

Des ore est drois que je me garde

De dire riens dont blasmez soie,

Quar cil qui une fois foloie

Se doit bien atant chastoier!

Des or me gart de foloier!

Si ferai je, si deüst faire

La desloiaux de put'afaire,

Pasiphé, qui trop foloia,

Quant onques son cuer aploia

A tel desverie penser. (OM, VIII, vv. 769-83)

Pour l'auteur de l'Ovide moralisé, prononcer un mot vulgaire effectue la liaison entre une infamie et la pensée qui la motive. C'est un moment quasi confessionnel qui explique peut-être l'affirmation que fait notre auteur, dans son épilogue, que le travail qu'il a mis à compléter cet ouvrage servira de "penitance des mesfais/Et des outrages que j'ai fais» (OM, XV, vv. 7469-70). Cette remarque nous rappelle également le rapprochement que fait Jean de Meun, dans le discours de Raison, entre l'obscénité de certains membres du corps et en même temps des mots qui les désignent, la bouche étant un site primaire de la transgression.

J'ai suggéré ailleurs que les aspects transgressifs du texte de Jean de Meun - son obscénité, ses jeux linguistiques, la structure dialogique de sa summa poétique - sont attribuables au désir de défier tout mouvement de censure motivée par une intolérance quelconque ${ }^{18}$. L'auteur de l'Ovide moralisé ne partage pas les intentions subversives de Jean de Meun, mais il est fasciné par la façon dont certaines images, mythiques ou autres, présentent des analogies avec le mystère chrétien, qui reste au fond inintelligible. À un certain moment, lorsqu'il parle de l'unité de Dieu, par contraste avec la diversité de sa création, il semble penser au principe qui préside à la démultiplication de son propre texte et de ses images : 
[...] Quanqu'a en la deïté

Tout est un, sans diverseté,

Mes quant a l'effait et a l'oeuvre

Que sapience et amours oeuvre,

Et quant a nostre cognoissance,

I samble il avoir differance. (OM, I, vv. 3275-3280)

Si notre connaissance ne peut procéder que par «differance», les efforts pour comprendre Dieu sont voués à se multiplier dans une suite d'images variées. D'où l'abîme qui sépare l'intention de l'auteur, celle de dévoiler la vérité des fables, et les effets de sa performance exégétique, qui restent profondément ancrés dans la lettre, dans la chair. Voilà pourquoi l'accusation de monologisme, basée sur la substitution d'un sens pour un récit, n'a qu'un rapport très limité avec ce texte. Car si le récit chrétien est le but manifeste de la quête de l'exégète, il semble bien que, ce mystère, l'Incarnation contre nature, aussi privilégié soit-il, soit lui-même encore une autre métamorphose, une autre mutacion qui nécessite sa propre glose. Ou plutôt, qui nécessite d'autres fables pour être intelligible. Selon l'auteur, les fables de l'Antiquité sont dangereuses, car les lire à la lettre attirerait l'accusation d'hérésie :

Et qui la fable ensi creroit

Estre voire, il meserreroit

Et seroit bogrerie aperte. (OM, XV, vv. 2533-35)

Les fables ne disparaissent pas, ne sont pas transformées une fois la glose appliquée. Au fur et à mesure de notre périple à travers ce vaste texte, l'articulation de la doctrine chrétienne, de ses dogmes pour le moins paradoxaux, finit par entrer dans une relation de dépendance avec ces mythes qui ne s'effacent point. Et à travers ce que j'ai appelé un effet de contamination, l'affinité de la métaphore fondatrice du christianisme avec les images conventionnelles du mariage et de la procréation hétérosexuels se trouve pour le moins atténuée, comme on le devine dans la citation suivante, également attachée à la fable d'Orphée :

Cil crierres de tout le monde

Fist jointure dou cors à l'ame

Et mariage d'ome à fame,

Mes l'une ne l'autre jointure

N'est ne si fine ne si pure

Que maint n'en soit puis mescheü

Qui puis ont maint encombre eü. (OM, X, vv. 457-63)

41 À travers les interstices du texte, on voir sourdre une autre affinité possible, cette soidisant perversité irrationnelle et non naturelle (défi donc des deux autorités clés de Jean de Meun, Raison et Nature!) des relations sexuelles qui sont par définition en défaut parce que, pour emprunter les paroles de l'auteur, elles n'ont aucun espoir d'une lignée. En défaut dans un sens, ces relations «contre nature » détiennent tout de même une efficacité métaphorique parce qu'elles démontent la différence des sexes dont l'accouplement, quelque «naturel» qu'il soit dans la définition médiévale, est foncièrement incapable de comprendre la jointure la plus centrale de toutes. 


\section{NOTES}

1.M.-R. Jung, "Les éditions manuscrites de l'Ovide moralisé », Cahiers d'Histoire des Littératures Romanes, 20, 1996, pp. 251-74 [251]. Sur les manuscrits et la réception de l'œuvre, voir également, du même auteur, «Ovide, texte, translateur et gloses dans les manuscrits de l'Ovide moralisé ", The Medieval Opus : Imitation, Rewriting, and Transmission in the French Tradition, éd. D. Kelly, Faux Titre, 116, Amsterdam/Atlanta, Rodopi, 1996, pp. 75-98.

2.La seule édition reste celle que fit publier Cornelis De Boer en cinq tomes, qui parurent entre 1915 et 1938 : Ovide moralisé, Verhandelingen der Koninklijke Akademie van Wetenschappen te Amsterdam, Afdeeling Letterkunde, Nieuwe Reeks, t.. 15, 21, 30 , 36-37, 43, Amsterdam, 1915-38. Toutes nos citations viennent de cette édition.

3.M.-R. Jung, "Ovide, texte, translateur », art. cit., pp. 77-8, se réfère au « titre moderne », qui selon lui correspond bien à certaines rubriques dans les manuscrits, telles que «les fables d'Ovide [...] translatees et ramenees a moralité », ou « les fables d'Ovide avec les exposicions et les alegories ».

4.Pour des détails sur la datation, voir Jung, «Les éditions manuscrites », pp. 253-55. 5.Nous citons d'après l'édition de A. Strubel, Guillaume de Lorris et Jean de Meun, Le Roman de la Rose, Paris, LGF, 1992. Pour l'influence de Jean de Meun sur l'Ovide moralisé, voir R. Copeland, Rhetoric, Hermeneutics and Translation in the Middle Ages: Academic Tradition and Vernacular Texts, Cambridge [U.K.], Cambride U. P., 1991, pp. 119-22. 6.Jung, ibid., pp. 254-55, fait remarquer que Clémence de Hongrie, épouse de Louis X, possédait un manuscrit de l'Ovide moralisé, selon l'inventaire de ses livres fait après sa mort en 1328, et que le plus ancien manuscrit qui nous soit parvenu est un manuscrit de luxe sûrement destiné à un patron noble. En effet, les compilations manuscrites de textes religieux en langue vernaculaire des XIII ${ }^{e}$ et $\mathrm{XIV}^{\mathrm{e}}$ siècles visaient souvent un public aristocratique féminin.

7. «Boethius'De Consolatione by Jean de Meun », éd. V. L. Dedeck-Héry, Mediaeval Studies, 14, 1952, p. 168.

8.Texte cité d'après BNF ms. lat. 14136.

9.Pour la question de la duplex causa efficiens - relation entre Dieu comme auteur ultime de la Bible et les auteurs humains qui l'ont réalisée -, que nous n'abordons pas ici mais qui est d'une réelle importance théorique, voir Copeland, op. cit., pp. 112-13.

10.R. Blumenfeld-Kosinski, Reading Myth, Stanford, Stanford U. P., 1997, p. 89

11.T. Cave, The Cornucopian Text, Oxford, Clarendon Press, 1979, pp. 95-6.

12.R. Tuve, Allegorical Imagery, Princeton, Princeton U. P., 1966, p. 294 (se référant spécifiquement à la compilation imprimée de Colart Mansion datant de 1484).

13. Nous citons d'après l'édition en quatre volumes de H. E. Butler, Cambridge [U.S.A.], Harvard University Press, 1958-60.

14.Nous citons d'après l'édition de J. Willis, Leipzig, Teubner, 1963.

15.Voir mon «Language and Dismemberment: Abelard, Origen and the Romance of the Rose ", Rethinking the Romance of the Rose : Text, Image, Reception, éd. Kevin Brownlee et Sylvia Huot, Philadelphie, University of Pennsylvania Press, 1992, pp. 101-30.

16.E. Langlois affirme en note (t. V, p. 110) : «J'ignore ce qu'est le jeu de marteaux, dont je n'ai rencontré aucune autre mention »; F. Lecoy remarque de son côté, «Pas plus que 
Langlois, je ne saurais dire quel est ce jeu de marteaux. » A. Strubel ne commente pas le passage.

17.Ce n'est pas dire que l'auteur ne parle jamais du masculin de manière générale, mais que dans l'exemple présent il fait basculer l'universel vers le singulier, et notamment dans la glose, où c'est plutôt le message universel qui devrait s'imposer. Il est donc intéressant de remarquer à cet égard que l'auteur parle du masculin lorsqu'il raconte la fable d'Orphée : «Foï toute amour femeline/Si se prist à la masculine » (OM, X, vv. 590-91).

18. «Words and Deeds : Jean de Meun's Romance of the Rose and the Hermeneutics of Censorship », New Literary History, 28, 2, 1997, pp. 345-66.

\section{AUTEUR}

DAVID F. HULT

Université de Californie - Berkeley 